Discrete Comput Geom 27:501-529 (2002)

DOI: $10.1007 / \mathrm{s} 00454-001-0077-0$

Geometry

\title{
Fractional Power Series Solutions for Systems of Equations
}

\author{
John McDonald \\ School of CTI, DePaul University, \\ Chicago, IL 60604, USA \\ jmcdonald@cs.depaul.edu
}

\begin{abstract}
In this paper we extend the explorations in [8] to include the fractional power series expansions of $k$ equations in $d$ variables, where $d>k$. An analog of Newton's polygon construction which uses the Minkowski sum $P$ of the Newton polytopes $P_{1}, \ldots, P_{k}$ of the $k$ equations is given for computing such series expansions. If the Newton polytopes of these equations are the same, then the common domains of convergence for the solutions correspond to the vertices of a certain fiber polytope $\Sigma(P)$. In general, our results suggest the existence of a "mixed fiber polytope" of $k$ polytopes. It is also indicated that there may be a relationship between these mixed fiber polytopes and a generalization of the discriminant, which we call the mixed discriminant.
\end{abstract}

\section{Introduction}

Suppose we have $k$ algebraic equations in $k+l$ variables:

$$
F_{i}\left(x_{1}, \ldots, x_{l}, y_{1}, \ldots, y_{k}\right)=0 \quad \text { for } \quad i=1, \ldots, k \text {. }
$$

We wish to construct $k$ fractional power series expansions, $y_{i}=\varphi_{i}\left(x_{1}, \ldots, x_{l}\right)$, for $i=1, \ldots, k$, such that formally $F_{j}\left(x_{1}, \ldots, x_{l}, \varphi_{1}, \ldots, \varphi_{k}\right)=0$ for all $j$. We consider the domains of covergence of such series expansions and investigate the complete sets of fractional power series solutions of these equations that converge in some common region of $\left(\mathbb{C}^{*}\right)^{l}$, where $\mathbb{C}^{*}=\mathbb{C}-\{0\}$.

Surprisingly, this classical setup, which dates back to Newton, is related to the concept of fiber polytopes as introduced in [3]. In the classical case of two variables and one equation, and in the case of $k$ equations in $k+1$ variables [4], the fiber polytopes involved are simply line segments. Newton's original construction can be found in [14].

In [8] we demonstrated the relationship in a more general setting ( $k=1$ and $l$ arbitrary). For computing such series expansions we gave an extension of Newton's construction, based on the Newton polytope of $F\left(x_{1}, \ldots, x_{l}, y_{1}\right)$. In that case, however, only 
a special class of fiber polytopes appeared, namely those that arise from the projection of a polytope to a line segment.

In this paper we consider the general case. As we are working with $k$ equations, we use the $k$ Newton polytopes $P\left(F_{i}\right) \subset \mathbb{R}^{k+l}$ of the $F_{i}$, and also consider the Minkowski sum $P$ of the $P\left(F_{i}\right)$. Whereas in Newton's original construction and in [8] we considered edges of the Newton polytope of $F$, the construction here is based on $k$-faces of $P$.

In the most general case we cannot assure that the construction actually gives a series solution. However, under certain explicit conditions we can prove that the construction can be carried out, and that the series built have common domains of convergence. These conditions are direct generalizations of the simple-root condition given for one equation in [8].

We will see that, generally, the number of series solutions converging in a given cone is equal to the mixed volumes of the projections of $P\left(F_{1}\right), \ldots, P\left(F_{k}\right)$ to $\mathbb{R}^{k}$. This agrees with the theorem due to Bernstein [2] on the number of solutions to a system of equations.

In the last section we show that, under the above conditions, the system of common domains of convergence of complete sets of solutions is closely related to the normal fan of a certain fiber polytope $\Sigma(P)$ of $P$. In general, this fan will be coarser than the normal fan of this fiber polytope, but if all of the $P\left(F_{i}\right)$ are equal, then the fans will be equal.

The existence of such a fan suggests the existence of a "mixed fiber polytope" $\Sigma\left(P_{1}, \ldots, P_{k}\right)$ of $k$ polytopes. This polytope should be a summand of the fiber polytope of their Minkowski sum, and should be equal to the fiber polytope $\Sigma(P)$ in the event that the $k$ polytopes are equal. Evidence for the existence of such a polytope is strengthened by the findings of Michiels and Cools in [9], where they built a mixed analog of the secondary polytope [5]. Finding such a polytope would conveniently generalize the relationship in [8] between power series expansions and the normal fans of polytopes. Moreover, the results here suggest a possible relationship between this mixed fiber polytope and a generalization of the classical discriminant, called the mixed discriminant.

\section{Preliminary Concepts}

\subsection{Polytopes}

Consider a real vector space $V=\mathbb{R}^{m}$. We use the standard definitions of a polytope, face and Minkowski sum, and include brief definitions here when necessary. For more information see [15].

Definition 2.1. A polytope $P \subset V$ is the convex hull of a finite subset of vertices in $V$. A polytope is called rational if all of its vertices are rational. Let $P$ be an $m$-dimensional polytope, and let $\gamma \in\left(\mathbb{R}^{m}\right)^{*}$ be a linear functional. Then the extreme face of $P$ in the direction $\gamma$ is the polytope

$$
f_{\gamma}=\left\{\mathbf{p} \in P:\langle\gamma, \mathbf{p}\rangle \geq\langle\boldsymbol{\gamma}, \mathbf{p}\rangle^{\prime} \text { for all } \mathbf{p}^{\prime} \in P\right\} ;
$$

$f_{\gamma}$ has a real dimension $k$ and is called a $k$-face of $P$. A vertex of $P$ is a face of dimension 0 , while a facet is an $(m-1)$-dimensional face. Through any $k$-face $f$ there passes a unique $k$-plane determined by $f$. 
A hyperplane $H \subset \mathbb{R}^{m}$ is said to support a polytope $P$ if $H \cap P \neq \emptyset$, and $P$ lies entirely in one of the half-spaces determined by $H$.

Definition 2.2. Let $P_{1}, \ldots, P_{n}$ be $n$ polytopes of dimension $m_{i}$. The Minkowski sum of these polytopes is the polytope of all vector sums of elements of $P_{1}, \ldots, P_{n}$. Let $f$ be a face of $P$. We say that $f$ is decomposable if $f=f_{1}+\cdots+f_{n}$ where $f_{i}$ is a face of $P_{i}$ and $\operatorname{dim}\left(f_{i}\right)>0$ for all $i$, otherwise $f$ is called indecomposable. For more on the decomposability of polytopes see [11].

Definition 2.3. Let $P_{1}, \ldots, P_{n}$ be $m$-dimensional polytopes. The mixed volume $\operatorname{Vol}\left(P_{1}\right.$, $\left.\ldots, P_{n}\right)$ is the alternating sum

$$
\operatorname{Vol}\left(P_{1}, \ldots, P_{n}\right)=\frac{1}{m !} \sum_{k=1}^{n}(-1)^{n-k} \sum_{1 \leq i_{1}<\cdots<i_{k} \leq n} \operatorname{Vol}\left(P_{i_{1}}+\cdots+P_{i_{k}}\right),
$$

where the volume $\operatorname{Vol}(P)$ of a polytope is normalized so that the standard $n$-simplex has volume 1. It is well known that $\operatorname{Vol}(P, \ldots, P)=\operatorname{Vol}(P)$.

\subsection{The Newton Polytope of a Polynomial}

The Newton polytope of a polynomial is key for connecting the study of polynomials and their power series expansions to convex geometry.

Definition 2.4. Let $F$ be a polynomial in $m$ variables, i.e.

$$
F=\sum_{I} a_{I} x^{I}
$$

with $I \in \mathbb{Q}^{m}$ ranging over some finite subset. The Newton polytope of $F$ is the rational polytope

$$
P(F)=\operatorname{conv}\left\{I \in \mathbb{Q}^{n}: a_{i} \neq 0\right\} .
$$

The set $S_{F}=\left\{I \in \mathbb{Q}^{m}: a_{I} \neq 0\right\}$ is called the support of $F$, also denoted $\operatorname{Supp}(F)$.

Let $F_{1}, \ldots, F_{k}$ be $k$ polynomials in $\ell$ variables. Let $S_{i} \subset \mathbb{R}^{\ell}$ be their supports. By considering the coefficients of these polynomials as variables, we can consider $F_{i}$ as a point in $\mathbb{C}^{S_{i}}$. Hence we can consider the system $F_{1}, \ldots, F_{k}$ as an element in the space $\Pi \mathbb{C}^{S_{i}}$. By a generic system of equations, we mean a system $F_{1}, \ldots, F_{k}$ which lies on some specific Zariski open subset of $\Pi \mathbb{C}^{S_{i}}$.

The following theorem is a direct consequence of Bernstein's theorem on the number of roots of a generic system of polynomials [2].

Theorem 2.1. Consider a generic system of $k$ equations in $\ell$ unknowns,

$$
F_{i}\left(y_{1}, \ldots, y_{\ell}\right)=0 \quad \text { for } \quad i=1, \ldots, k
$$


and let $P_{i}=P\left(F_{i}\right)=\operatorname{conv}\left(S_{i}\right)$ be the Newton polytope of $F_{i}$ for all $i$. Then the number of power series solutions of this system converging in a given region is equal to the mixed volume $\operatorname{Vol}\left(P_{1}, \ldots, P_{k}\right)$ of the polytopes $P_{1}, \ldots, P_{k}$.

\subsection{Convex Polyhedral Cones}

A convex polyhedral cone in $\mathbb{R}^{m}$ is a set of the form

$$
C=\left\{r_{1} \mathbf{v}_{1}+\cdots+r_{n} \mathbf{v}_{n}: r_{i} \in \mathbb{R}, r_{i} \geq 0\right\},
$$

where $\mathbf{v}_{1}, \ldots, \mathbf{v}_{n} \in \mathbb{R}^{m}$ are fixed vectors. A cone is rational if $\mathbf{v}_{i} \in \mathbb{Q}^{m}$ for every $i$, and is strongly convex if it contains no non-trivial linear subspaces.

We identify the dual space $\left(\mathbb{R}^{m}\right)^{*}$ with $\mathbb{R}^{m}$, by means of the usual pairing $\langle\mathbf{u}, \mathbf{x}\rangle=$ $\sum u_{i} x_{i}$. Let $C$ be a strongly convex rational polyhedral cone in $\mathbb{R}^{m}$. Define the dual cone, $C^{*} \subset\left(\mathbb{R}^{m}\right)^{*}$, to be the set

$$
C^{*}=\left\{\mathbf{u} \in \mathbb{R}^{m}:\langle\mathbf{u}, \mathbf{x}\rangle \leq 0, \forall x \in C\right\} .
$$

This is the cone consisting of all linear functionals which have a maximum on $V$. In the following we do not use bold-face to denote elements of $C^{*}$. This is to distinguish elements of the dual from vectors in $\mathbb{R}^{m}$.

We will often work with polytopes in $\mathbb{R}^{m}=\mathbb{R}^{k+\ell}$ where $k$ is the number of equations and $\ell$ is the number of independent variables. Therefore, we assume throughout this work that we have chosen a direct sum decomposition

$$
\mathbb{R}^{m}=\mathbb{R}^{\ell} \oplus \mathbb{R}^{k}
$$

The coordinates in $\mathbb{R}^{\ell}$ and $\mathbb{R}^{k}$ will be denoted by $\alpha_{1}, \ldots, \alpha_{\ell}$ and $\beta_{1}, \ldots, \beta_{k}$, respectively.

Definition 2.5. Let $\Pi$ be a $k$-plane in $\mathbb{R}^{k+\ell}$, then $\Pi$ is called admissible if the projection $\pi: \Pi \rightarrow \mathbb{R}^{k}$ is injective. Thus on such a $\Pi$ we have a parameterization

$$
\alpha_{i}=\sum_{i}\left(\delta_{i j} \beta_{j}+\varepsilon_{i}\right)=\varepsilon+\sum_{i}\left(\delta_{i j} \beta_{j}\right)
$$

The matrix $\left\|\delta_{i j}\right\|$ is called the matrix of slopes of $\Pi$. On a polytope $P$ we say that a $k$-face is admissible if the $k$-plane it determines is admissible.

Definition 2.6. Let $f$ be an admissible face of $P$, and let $w \in\left(\mathbb{R}^{\ell}\right)^{*}$ be a linear functional. Then $w$ determines a unique hyperplane $H_{f, w}$ in $\mathbb{R}^{m}$ which contains $f$ and on which $w$ is constant on every fiber of the projection to $\mathbb{R}^{k}$. (The equation $\langle w, x\rangle=z$ determines a hyperplane in $\mathbb{R}^{\ell}$.) Such a hyperplane is called $w$-constant.

Consider the case $k=1$. Let $w$ be any linear function on $\mathbb{R}^{\ell}$. We extend $w$ trivially to a linear function of $\mathbb{R}^{\ell+1}$ by defining, for $\mathbf{x} \in \mathbb{R}^{\ell+1}, \mathbf{x}=\left(x_{0}, \ldots, x_{\ell}\right)$, the action of $w$ on $\mathbf{x}$ to be

$$
\langle w, \mathbf{x}\rangle:=\left\langle w,\left(x_{1}, \ldots, x_{\ell}\right)\right\rangle .
$$


A hyperplane $H$ in $\mathbb{R}^{\ell+1}$ is then $w$-constant if for each $c \in \mathbb{R}$,

$$
\left\langle w, H \cap\left\{x_{\ell+1}=c\right\}\right\rangle:=\left\{\langle\mathbf{w}, x\rangle: \mathbf{x} \in H \cap\left\{x_{\ell+1}=c\right\}\right\}=\left\{d_{c}\right\}
$$

for some $d_{c} \in \mathbb{R}$, i.e. $w$ is constant on each "vertical" section of $H$. Since we will be using the $\left(x_{1}, \ldots, x_{\ell}\right)$-hyperplane frequently, we call it the null-hyperplane.

\subsection{Normal and Barrier Cones}

Let $w \in\left(\mathbb{R}^{\ell}\right)^{*}$ be a linear functional on $\mathbb{R}^{\ell}$ such that the coordinates of $w$ are linearly independent over $\mathbb{Q}$. Such a linear functional is called irrational. The equation $\langle w, \mathbf{x}\rangle=z$ for any fixed $z$ has at most one solution in $\mathbb{Q}^{\ell}$. Therefore, $w$ induces a linear ordering on $\mathbb{Q}^{\ell}$.

Consider the projection

$$
\pi: P \longrightarrow Q=\pi(P) \subset \mathbb{R}^{k}
$$

onto the last $k$ coordinates. The fiber of $\pi$ over any interior point $q \in Q$ is a $(d-k)$ dimensional polytope. Note that this fiber is given by a system of linear inequalities with rational coefficients, but possibly with irrational right-hand sides. Since $w$ is irrational, there exists a unique point $\mathbf{p}_{q} \in \pi^{-1}(q)$ such that

$$
\left\langle w, \mathbf{p}_{q}\right\rangle>\langle w, \mathbf{p}\rangle \quad \text { for all } \mathbf{p} \in \pi^{-1}(q) .
$$

Therefore, $w$ defines a section of $\pi$ which is called the maximal section of $\pi$ with respect to $w$. This section is denoted by $\mathcal{S}_{w, \pi}(P)=\mathcal{S}_{w}(P)$.

Definition 2.7. Let $P$ be an $m$-dimensional polytope, and let $f$ be an admissible $k$-face of $P$, with $m=k+\ell$. The normal cone of $f$ is the closure of the set of all irrational linear functionals in $\left(\mathbb{R}^{\ell}\right)^{*}$ such that $f$ is contained in $\mathcal{S}_{w, \pi}(P)$. The normal cone of $f$ is denoted by $N(f)$. The barrier cone, denoted $B(f)$, is defined to be the dual $N^{*}(f)$ of $N(f)$.

In particular, this means that for a vertex $\mathbf{v}$ of $P$, the normal cone $N(\mathbf{v})$ is the cone in $\left(\mathbb{R}^{m}\right)^{*}$ consisting of all linear functionals which achieve a maximum on $P$ at $\mathbf{v}$. Notice that, under this definition, the barrier cone of a $k$-face does not lie in the same space as the polytope. Rather, it lies in $\mathbb{R}^{\ell}$. Likewise, the normal cone of a $k$-face lies in $\left(\mathbb{R}^{\ell}\right)^{*}$ rather than $\left(\mathbb{R}^{m}\right)^{*}$.

There is another, more geometric, definition of the barrier cone of a vertex $\mathbf{v}$ :

$$
C(\mathbf{v})=\left\{\lambda(\mathbf{p}-\mathbf{v}): \lambda \in \mathbb{R}_{+}, \mathbf{p} \in P(F)\right\},
$$

i.e. the cone spanned by the vectors from $\mathbf{v}$ to points in $P(F)$. The dual of $C(\mathbf{v})$ is the normal cone of $\mathbf{v}$.

Definition 2.8. Let $K(f)$ be the $k$-plane in $\mathbb{R}^{m}$ which contains the face $f$. The set

$$
N(f) \times K(f):=\left\{\mathbf{z} \in \mathbb{R}^{m}=\mathbb{R}^{\ell} \oplus \mathbb{R}^{k}: \mathbf{z} \in N(f)+\mathbf{y}, \text { where } \mathbf{y} \in K(f)\right\}
$$

is called the barrier wedge of $f$, and is denoted by $W(f)$. The barrier wedge of a $k$-face is simply the cone spanned by all vectors from an interior point of $f$ to points in $P$. 


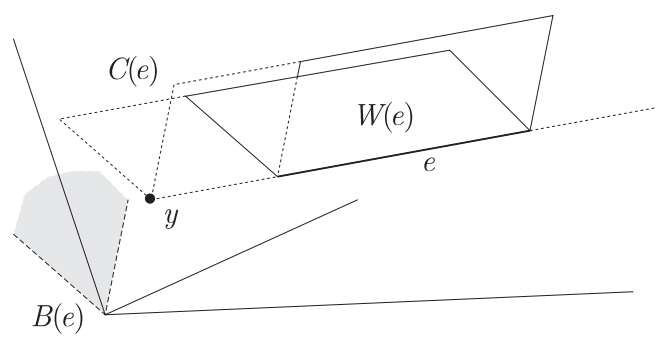

Fig. 1. The barrier wedge and cone associated to an edge.

Example 2.1. Let $e$ be any admissible edge of $P(F)$. The vertices of $e$ with the largest and smallest $x_{\ell+1}$ coordinates are respectively called the major and minor vertices of $e$, and are denoted by $m(e)$ and $M(e)$, respectively. Write $m(e)=\left(p_{1}, \ldots, p_{\ell+1}\right)$ and $M(e)=\left(q_{1}, \ldots, q_{\ell+1}\right)$. The slope vector $S(e)$ of $e$ with respect to $x_{\ell+1}$ is

$$
S(e)=\frac{1}{q_{\ell+1}-p_{\ell+1}}\left(q_{1}-p_{1}, \ldots, q_{\ell}-p_{\ell}\right) .
$$

For such an edge, the barrier cone of $e$ can be described as follows:

Let $L$ be the line in $\mathbb{R}^{\ell+1}$ determined by $e$, and let $\mathbf{y}$ be the point of intersection of $L$ with the null-hyperplane (such a point exists since $e$ was assumed not to be parallel to this plane). Then the barrier wedge of $e$ in $\mathbb{R}^{\ell+1}$ is

$$
W(e)=\left\{\lambda(\mathbf{p}-\mathbf{x})+\mathbf{x}: \lambda \in \mathbb{R}_{+}, \mathbf{p} \in P(F), \mathbf{x} \in L\right\} .
$$

The intersection of this wedge with the null-hyperplane is a convex rational polyhedral cone, $C(e)=B(e)+\mathbf{y}$, which has its vertex at $\mathbf{y}$. See Fig. 1. This is a translate of the barrier cone $B(e)$. For convenience, we often relax our definition and refer to translates of cones as cones themselves.

\subsection{The Normal Fan}

One important aspect of the normal cones of the vertices of a polytope is that they knit together to form a fan of $\left(\mathbb{R}^{m}\right)^{*}$, since every linear functional attains a maximum on some face of $P$, and hence at some vertex of $P$.

Definition 2.9. Let $\left\{\mathbf{v}_{\mathbf{1}}, \ldots, \mathbf{v}_{\mathbf{n}}\right\}$ be the set of vertices of $P$. Then the collection of pairwise disjoint cones $N\left(\mathbf{v}_{1}\right), \ldots, N\left(\mathbf{v}_{n}\right)$ forms a fan that covers $\left(\mathbb{R}^{m}\right)^{*}$. This fan is called the normal fan of $P$ and is denoted $\Delta_{P}$. These definitions coincide with the standard notions [15].

As an example of how the structure of the Minkowski sum of a collection of polytopes is related to their individual structures, notice that the normal fan of the Minkowski sum of $n$ polytopes $\Delta_{P_{1}+\cdots+P_{n}}$ is the smallest common refinement of $\Delta_{P_{1}}, \ldots, \Delta_{P_{n}}$. That is, cones in the normal fan of the Minkowski sum are intersections of cones in the 
normal fans of each of the summands. For a more complete discussion of these ideas see pp. 190-191 of [5] and see [15].

\subsection{Fiber Polytopes}

The applications of the techniques in this paper use the notion of the fiber polytope of a projection of two polytopes $P \stackrel{\psi}{\longrightarrow} Q$ as defined in [3], for a projection $\psi$ from $X$ to $Y$. Let us recall the definitions.

Let $P \subset \mathbb{R}^{N}$ be a convex polytope. Let $\psi: \mathbb{R}^{N} \rightarrow \mathbb{R}^{M}$ be a surjective linear map and let $Q=\psi(P)$. The Minkowski integral is the set of vector integrals

$$
\int P=\int_{Q} P=\int_{Q} \gamma(x) d x,
$$

where $\gamma$ ranges over all continuous sections of $\psi$.

The fiber polytope $\Sigma_{\psi}(P, Q)$ is defined to be the normalized Minkowski integral

$$
\Sigma(P, Q):=\frac{1}{\operatorname{Vol}(Q)} \int_{Q} P .
$$

The following are some of the important properties and results concerning fiber polytopes. We leave out most of the proofs here, as they can be found in [3] or [15]. Two polytopes are called normally equivalent if they have the same normal fan.

Proposition 2.2. The fiber polytope $\Sigma(P, Q)$ is a non-empty convex polytope in $R^{N-M}$. Moreover, there exists a finite subset $\left\{x_{1}, \ldots, x_{n}\right\} \subset Q$ such that the Minkowski sum of the fibers $P_{x_{1}}+\cdots+P_{x_{n}}$ is normally equivalent to $\Sigma(P, Q)$.

Let $\psi: P \longrightarrow Q$ and let $F \subset P$ such that $F_{x}$ is a face of $P_{x}$ for every $x$ in $Q$. Then the projection $F \longrightarrow Q$ is called a face bundle of $P$. If there exists a linear functional $\psi$ on $R^{N-M}$ such that $F_{x}$ is extreme in the direction $\psi$ (in the same sense as above), then $F$ is called a coherent face bundle. Notice that a coherent face bundle such that each $F_{x}$ consists of a single point (i.e. a vertex of $P_{x}$ ) is a maximal section of $P \longrightarrow Q$ in some direction.

Proposition 2.3. The faces of $\Sigma(P, Q)$ are in one-to-one correspondence with the coherentface bundles of $P$. In fact, the faces of the Minkowski integral $\int_{Q} P$ are the integrals of the coherent face bundles of $P$. In particular, the vertices of $\Sigma(P, Q)$ correspond to the maximal sections of $P \longrightarrow Q$.

Putting these first two propositions together yields

Corollary 2.4. Let $v$ be a vertex of $\Sigma(P, Q)$, and let $\varphi$ be the corresponding maximal section of $P \longrightarrow Q$. The normal cone of $v$ is the intersection of the normal cones of the $\varphi(x) \subset P_{x}$. Equivalently, the normal cone of $v$ is the intersection of the normal cones of the $M$-faces of $P$ in $\varphi(Q)$. 


\subsection{Rings of Fractional Power Series}

The following definitions will facilitate our connections between the classical techniques in the previous section with series expansions in several variables.

If $n$ is an integer greater than zero, and $C$ is a strongly convex rational polyhedral cone in $\mathbb{R}^{\ell}$, then the set

$$
C_{n}=C \cap \frac{1}{n} \mathbb{Z}^{\ell}
$$

forms a semigroup under addition. From such a semigroup we can form the semigroup ring $\mathbb{C}\left[C_{n}\right]$, i.e. the ring of all finite formal sums of the form $\sum a_{\alpha} x^{\alpha}$ where $\alpha \in C_{n}$. We regard elements of $\mathbb{C}\left[C_{n}\right]$ as fractional Laurent polynomials in the variables $x_{1}, \ldots, x_{\ell}$. Let $\mathbb{C}\left[\left[C_{n}\right]\right]$ be the completion of the ring $\mathbb{C}\left[C_{n}\right]$, the ring of all formal fractional power series, $\sum_{\alpha \in C_{n}} a_{\alpha} x^{\alpha}$.

Definition 2.10. If $C$ is a strongly convex rational polyhedral cone in $\mathbb{R}^{\ell}$, then the ring of fractional power series in the variables $x_{1}, \ldots, x_{\ell}$ with support in $\mathrm{C}$ is defined by

$$
\mathbb{C}\left[\left[C_{\mathbb{Q}}\right]\right]=\bigcup_{n=1}^{\infty} \mathbb{C}\left[\left[C_{n}\right]\right] .
$$

More generally, the ring of fractional power series with support in some translate of $C$ is

$$
\mathbb{C}\left(\left(C_{\mathbb{Q}}\right)\right)=\bigcup_{\alpha \in \mathbb{Q}^{\ell}} x^{\alpha} \mathbb{C}\left[\left[C_{\mathbb{Q}}\right]\right]
$$

It is essential to require that $C$ be strongly convex, otherwise the set $\mathbb{C}\left[\left[C_{\mathbb{Q}}\right]\right]$ does not have a well-defined multiplicative structure, since finding a coefficient when multiplying two such series would involve an infinite sum.

Let $C$ be a strongly convex rational polyhedral cone. For any

$$
f(x)=\sum_{\alpha \in \mathbb{Q}^{\ell}} a_{\alpha} x^{\alpha}
$$

in $\mathbb{C}\left(\left(C_{\mathbb{Q}}\right)\right)$ we define the support of $f$ to be the set of exponents which appear in $f$, i.e. $\operatorname{Supp}(f)=\left\{\alpha \in \mathbb{Q}^{\ell}: a_{\alpha} \neq 0\right\}$. Since $f \in x^{\alpha} \mathbb{C}\left[\left[C_{n}\right]\right]$ for some $n$, the support of $f$ must lie in some lattice $(1 / n) \mathbb{Z}^{\ell}$.

\subsection{Convergence and Convex Geometry}

In order to speak of the convergence of fractional power series in $\mathbb{C}\left(\left(C_{\mathbb{Q}}\right)\right)$, we must define the manner in which these series act as functions on $\mathbb{C}^{N}$. More precisely, we must define the action of $x^{\alpha}=x_{1}^{\alpha_{1}} \cdots x_{\ell}^{\alpha_{\ell}}$ on $\left(\mathbb{C}^{*}\right)^{\ell}$. To do this we only need to choose, in each variable, a sector in $\mathbb{C}^{*}$ and define a branch of the logarithm in this sector, e.g. the principal branch of the log: let $\mathbb{C} \backslash \mathbb{R}_{-}$be the chosen sector and define

$$
x_{i}^{\alpha_{i}}=e^{\alpha_{i} \log x_{i}}
$$


for each variable $x_{i}$. We are primarily interested in the regions for which an $f \in \mathbb{C}[[C]]$ is absolutely convergent (i.e. where $\sum\left|a_{\alpha}\right||x|^{\alpha}$ converges).

Definition 2.11. If $C$ is a convex rational polyhedral cone, then $\mathbb{C}\left\{\left\{C_{\mathbb{Q}}\right\}\right\}$ will denote the subring of $\mathbb{C}\left(\left(C_{\mathbb{Q}}\right)\right)$ consisting of all series which are convergent at some point of $\left(\mathbb{C}^{*}\right)^{\ell}$, i.e. if for $f \in \mathbb{C}\left(\left(C_{\mathbb{Q}}\right)\right), D_{f}$ is the domain of convergence of $f$, then

$$
\mathbb{C}\left\{\left\{C_{\mathbb{Q}}\right\}\right\}=\left\{f \in \mathbb{C}\left(\left(C_{\mathbb{Q}}\right)\right): D_{f} \neq \emptyset\right\} .
$$

Note that $\mathbb{C}\left\{\left\{C_{\mathbb{Q}}\right\}\right\}$ consists only of convergent series whose exponents all lie in $\mathbb{Z}[1 / n]$ for some $\mathrm{n}$.

It is convenient to pass to the logarithms of the $\left|x_{i}\right|$ when considering convergence, therefore we introduce the space $\mathbb{R}_{\log }^{N}$ called the logarithmic space of $\left(\mathbb{C}^{*}\right)^{\ell}$, which is associated to $\left(\mathbb{C}^{*}\right)^{\ell}$ via the map

$$
\log \left(x_{1}, \ldots, x_{\ell}\right)=\left(\log \left(\left|x_{1}\right|\right), \ldots, \log \left(\left|x_{\ell}\right|\right)\right)
$$

The usefulness of these notations is indicated by the following lemmas. For their proofs see [8].

Lemma 2.5. For each $f \in \mathbb{C}\left\{\left\{C_{\mathbb{Q}}\right\}\right\}$ the domain of convergence of $f$ has the form $\log ^{-1}(U)$, for some convex set $U \subset \mathbb{R}_{\log }^{\ell}$.

Lemma 2.6. Suppose $f=\sum a_{\alpha} x^{\alpha}$ is in $\mathbb{C}\left(\left(C_{\mathbb{Q}}\right)\right)$ and $f$ has a non-empty domain of convergence $D$ (i.e. $f \in \mathbb{C}\left\{\left\{C_{\mathbb{Q}}\right\}\right\}$ ), then there exists some $A \in\left(\mathbb{C}^{*}\right)^{\ell}$ such that $\left|a_{\alpha}\right| \leq\left|A^{\alpha}\right|$ for almost all $\alpha$. Moreover, if $x$ is any point in $D$, and $C$ is any cone which contains the Newton polytope $P(f)$, then $C^{*}+\log (x) \subset \log (D)$.

We say that $f$ converges at some point $y \in \mathbb{R}_{\log }^{N}$ if $\log ^{-1}(y) \subset D$ where $D$ is the domain of convergence for $f$. The above lemma can be summarized by saying that if $f$ converges at some point $y \in \mathbb{R}_{\log }^{N}$, then $f$ converges on some translate of $C^{*}$. The following theorem is a direct result of these two lemmas, see [1] or [8].

Theorem 2.7. If $C$ is a cone in $\mathbb{R}^{\ell}$ and if a series $f \in \mathbb{C}\left(\left(C_{\mathbb{Q}}\right)\right)$ is algebraic over $\mathbb{C}\left[x_{1}, \ldots, x_{\ell}\right]$, then there is some translate of $C^{*}$ on which $f$ is convergent.

For more information on such topics, see [7].

\subsection{Transfinities and Transfinite Induction}

The construction of these fractional power series solutions is based on a transfinite algorithm similar to the methods used in constructing series solutions for polynomials over fields of characteristic $p$, see [10], [12] and [13]. Therefore, a brief review of transfinites and transfinite induction is in order, for more information, see [6]. 
Recall that a transfinite symbol, $\gamma$, is defined to be an equivalence class of well-ordered sets, where the equivalence is given by order-preserving bijection. Since bijections preserve the cardinality of a set, all sets in the equivalence class, $\gamma$, have the same cardinality. We call a transfinite countable if every set in its class is countable. Let $\Gamma$ denote the set of all countable transfinites, and note that $\Gamma$ is itself a well-ordered but uncountable set.

In $\Gamma$ there are two types of transfinites, those that arise as the immediate successor of a given $\gamma \in \Gamma$, and those that arise as the limit of the transfinites preceding it in the order on $\Gamma$. These two types are usually referred to as isolated and limit transfinites respectively and are written as

$$
\gamma+1 \text { and } \lim _{\delta<\gamma} \delta .
$$

For example, the empty set is a well-ordered set represented by the symbol 0 , it is the first ordinal number and is therefore the smallest symbol in $\Gamma$ under its well ordering. Any other finite symbol $n$ is the immediate successor of another symbol, namely $n-1$, and has $n+1$ as an immediate successor. Therefore all finite symbols are isolated transfinites. The first limit transfinite $\omega$ is the class which contains the well-ordered set of positive integers under their usual order. So, $\omega$ can be defined as

$$
\omega=\lim n \text {. }
$$

The isolated symbol $\omega+1$ is represented by sets which increase to a limit point and then contain either the limit point or some element larger than the limit point. For example, the set

$$
\left\{0, \frac{1}{2}, 1-\frac{1}{3}, 1-\frac{1}{4}, \ldots, 1\right\}
$$

is a set in the equivalence class $\omega+1$.

Transfinite induction is carried out in two steps: that of proceeding from $\gamma$ to $\gamma+1$, and that of passing to a limit transfinite $\gamma$, once the process has been carried out for all $\delta<\gamma$. For any $\gamma \in \Gamma$ we will build a series $\varphi_{\gamma}$ such that if $\gamma^{\prime}<\gamma$, then $\varphi_{\gamma^{\prime}}$ is a summand of $\varphi_{\gamma}$. We will accomplish this by showing that if we have built $\varphi_{\delta}$ for all transfinites $\delta<\gamma$, then we can build $\varphi_{\gamma}$. In particular this means that for an isolated transfinite, $\gamma$, if we have constructed $\varphi_{\gamma}$, then we can construct $\varphi_{\gamma+1}$. If $\gamma$ is a limit transfinite we must show that if we have $\varphi_{\delta}$ for all $\delta<\gamma$, then we can construct $\varphi_{\gamma}=\varphi_{\lim \delta}$. Finally, we must show that we can build $\varphi_{0}$ and that the process will stop after some countable transfinite $\gamma$.

Transfinite induction will only be formally needed in the following construction. Once we have the general construction for the series expansions, we will be able to prove that its exponents never actually accumulate. In practice, therefore, the construction is never transfinite in nature, and the series thus constructed are just ordinary fractional power series in several variables.

\section{The Construction for Generic Systems}

\subsection{The First Step}

Let

$$
F_{i}(x, y)=F_{i}\left(x_{1}, \ldots, x_{\ell}, y_{1}, \ldots, y_{k}\right)=0, \quad i=1, \ldots, k,
$$


be a system of $k$ equations in $k+\ell$ variables. We denote by $P_{i} \subset \mathbb{R}^{m}$ their Newton polytopes and appeal to the notation of Section 2.3 with respect to the decomposition of $\mathbb{R}^{m}=\mathbb{R}^{k+\ell}=\mathbb{R}^{\ell} \oplus \mathbb{R}^{k}$. We continue to use the notation of $\alpha$ and $\beta$ as the coordinates on $\mathbb{R}^{\ell}$ and $\mathbb{R}^{k}$, respectively. We assume, as before, that we have fixed an irrational linear functional $w \in\left(\mathbb{R}^{\ell}\right)^{*}$.

Suppose

$$
F_{i}(x, y)=\sum_{(\alpha, \beta) \in S_{i}} a_{i, \alpha, \beta} x^{\alpha} y^{\beta},
$$

where $S_{i}$ is the support of $F_{i}$ in $\mathbb{R}^{m}$. We need to build $k$ solution series expansions $y_{i}=\varphi_{i}\left(x_{1}, \ldots, x_{l}\right)$ of the form

$$
\varphi_{i}=\sum_{\gamma \in \Gamma} c_{\gamma, i} x^{\delta_{\gamma, i}}=\sum_{\gamma \in \Gamma} \psi_{\gamma, i}
$$

where $\Gamma$ is some well-ordered countable set, and for each $i$, the set $\left\{\delta_{\gamma, i}\right\}$, is a wellordered subset of $\mathbb{R}^{\ell}$ with respect to the order given by $w$. We denote by $\varphi_{\gamma, i}$ the $\gamma$ th partial sum of $\varphi_{i}$.

We first build all of the $\varphi_{1, i}$ and then we consider the inductive step of moving the construction from $\gamma$ to $\gamma+1$ and then define $\psi_{\gamma, i}$ for a limit transfinite $\gamma$. In many respects the first two parts are identical.

Let $P_{1}^{(0)}, \ldots, P_{k}^{(0)} \subset \mathbb{R}^{m}$ be the Newton polytopes of $F_{1}, \ldots, F_{k}$ (i.e. $P_{i}^{(0)}=P_{i}$ for all $i$ ) and let $P^{(0)}=P_{1}^{(0)}+\cdots+P_{k}^{(0)}$ be their Minkowski sum. For the purposes of iterating the process described below, we now write $F_{i}^{(0)}$ instead of $F_{i}$.

Let $\mathcal{S}$ be the section of $\pi: P^{(0)} \rightarrow \mathbb{R}^{k}$ determined by maximality with respect to $w$. We choose any admissible decomposable $k$-face $f^{(0)}$ of $\mathcal{S}$. Let $\left\{f_{i}^{(0)}\right\}$ be the faces of $P_{i}$ respectively which sum to $f^{(0)}$. We assume that $\operatorname{dim}\left(f_{i}^{(0)}\right)>0$ for all $i$, since if $f_{i}^{(0)}$ is a point, then the system would admit no solutions with all components different from zero. Let $Q^{(0)}$ be the $k$-plane determined by $f^{(0)}$ and let $Q_{i}^{(0)}$ be the translates of $Q^{(0)}$ which contain $f_{i}^{(0)}$, respectively, i.e. $P_{i}^{(0)} \cap Q_{i}^{(0)}=f_{i}^{(0)}$.

Since $f$ is admissible, it has a matrix of slopes

$$
\delta=\left[\begin{array}{c}
\delta_{1} \\
\delta_{2} \\
\vdots \\
\delta_{k}
\end{array}\right]=\left[\begin{array}{cccc}
\delta_{1,1} & \delta_{1,2} & \cdots & \delta_{1, \ell} \\
\delta_{2,1} & \delta_{2,2} & \cdots & \delta_{2, \ell} \\
\vdots & \vdots & \ddots & \vdots \\
\delta_{k, 1} & \delta_{k, 2} & \cdots & \delta_{k, \ell}
\end{array}\right] .
$$

For the first terms of our expansions we set

$$
\psi_{1, i}=c_{i} x_{1}^{-\delta_{i, 1}} \cdots x_{\ell}^{-\delta_{i, \ell}}
$$

where $c=\left(c_{1}, \ldots, c_{k}\right)$ is a non-zero solution to the system of equations

$$
\sum_{(\alpha, \beta) \in S_{i} \cap Q_{i}^{(0)}} a_{i, \alpha, \beta} c^{\beta}=0 .
$$


These equations are called the face equations of $f^{(0)}$. Note that by Theorem 2.1 the number of such solutions counting multiplicity is, in general, equal to the mixed volume of the projections of $f_{1}^{(0)}, \ldots, f_{k}^{(0)}$ to $\mathbb{R}^{k}$.

A priori we have no guarantee that this system actually has a solution. If all of the Newton polytopes of the $f_{i}^{(0)}$ are spanned by exactly two vertices, then the system will have exactly as many solutions as the mixed volume of their Newton polytopes. For the next part of the construction we assume it does have a solution. In Section 4 we give another explicit situation in which we can assure that the above system of equations actually has a solution.

A small point of notation: so as to make the following equations as presentable as they possibly can be, we forgo the use of bold-face. You may recognize the vector terms by their lack of an $i$ subscript.

\subsection{The Inductive Step}

Next, we look at the inductive step of proceeding from the transfinite $\gamma$ to the transfinite $\gamma+1$. We consider the case of a limit transfinite at the end of this section. So, assume that we have constructed $\varphi_{\gamma^{\prime}, i}$ for all $i$ and for all $\gamma^{\prime} \leq \gamma$, where $\gamma$ is an isolated transfinite symbol.

Suppose that, in constructing the $\varphi_{\gamma^{\prime}, i}$, we have built polynomials $F_{i}^{\left(\gamma^{\prime}\right)}$ and their polytopes $P_{i}^{\left(\gamma^{\prime}\right)}$. Let

$$
F_{i}^{\left(\gamma^{\prime}\right)}(x, y)=\sum_{(\alpha, \beta) \in S_{i}^{\left(\gamma^{\prime}\right)}} a_{i, \alpha, \beta}^{\left(\gamma^{\prime}\right)} x^{\alpha} y^{\beta},
$$

where $S_{i}^{\left(\gamma^{\prime}\right)}$ is the support of $F_{i}^{\left(\gamma^{\prime}\right)}$.

Suppose that we obtained faces $f_{i}^{\left(\gamma^{\prime}\right)}$ that sum to an admissible decomposable $k$-face $f^{\left(\gamma^{\prime}\right)}$ of their Minkowski sum $P^{\left(\gamma^{\prime}\right)}$. Also assume that

$$
\psi_{\gamma^{\prime}, i}=c_{i}^{\left(\gamma^{\prime}\right)} x^{-\delta_{i}^{\left(\gamma^{\prime}\right)}}
$$

where

$$
\left[\begin{array}{c}
\delta_{1}^{\left(\gamma^{\prime}\right)} \\
\delta_{2}^{\left(\gamma^{\prime}\right)} \\
\vdots \\
\delta_{k}^{\left(\gamma^{\prime}\right)}
\end{array}\right]
$$

is the slope matrix of $f^{\left(\gamma^{\prime}\right)}$, and $c^{\left(\gamma^{\prime}\right)}$ is a non-zero solution of its face equations, which have the same form as the face equations given above for $f^{(0)}$. Notice that all of these criteria are met by the objects used in the first step of the construction.

To proceed from $\gamma$ to $\gamma+1$ we substitute

$$
c_{1}^{(\gamma)} x^{-\delta_{1}^{(\gamma)}}+y_{1}, \ldots, c_{k}^{(\gamma)} x^{-\delta_{k}^{(\gamma)}}+y_{k}
$$


in place of $y_{1}, \ldots, y_{k}$ in the polynomials $F_{i}^{(\gamma)}$ to obtain the polynomials $F_{i}^{(\gamma+1)}$. Let $P_{i}^{(\gamma+1)}=P\left(F_{i}^{(\gamma+1)}\right)$ be their Newton polytopes and let $P^{(\gamma+1)}$ be the Minkowski sum of these polytopes. Let $S_{i}^{(\gamma+1)}$ be the support of $F_{i}^{(\gamma+1)}$. To continue this construction we need to find a face $f^{(\gamma+1)}$ on $P^{(\gamma+1)}$ satisfying the following three conditions:

1. If

$$
\left[\begin{array}{c}
\delta_{1}^{(\gamma+1)} \\
\delta_{2}^{(\gamma+1)} \\
\vdots \\
\delta_{k}^{(\gamma+1)}
\end{array}\right]
$$

is the matrix of slopes of $f^{(\gamma+1)}$ then $\left\langle w, \delta_{i}^{(\gamma+1)}\right\rangle>\left\langle w, \delta_{i}^{(\gamma)}\right\rangle$ for all $i$.

2. If $N\left(f^{(\gamma)}\right)$ is the normal cone of $f_{i}^{(\gamma)}$ (and likewise for $f^{(\gamma+1)}$ ) then

$$
N\left(f^{(\gamma+1)}\right) \cap N\left(f^{(\gamma)}\right) \neq \varnothing
$$

and in fact this intersection contains $w$.

3. The face equations of $f^{(\gamma+1)}$ have a non-zero solution.

We will find that it is not always possible to find such a face, and that, even when one exists, its face equations may have no solutions. In Section 4 we explore a case in which we can always find a decomposable face satisfying all three of these criteria.

For the rest of this section assume that, at every isolated transfinite symbol $\gamma+1$ such that the expansions $\varphi_{\gamma, i}=\sum_{\gamma^{\prime} \leq \gamma} \psi_{\gamma^{\prime}, i}$ are not a solution to the original system of equations, we can find a face of $P^{(\gamma+1)}$ satisfying the above conditions. Then we set, as in the first step of the construction,

$$
\psi_{\gamma+1, i}=c_{i}^{(\gamma+1)} x^{-\delta_{i}^{(\gamma+1)}},
$$

where $c^{(\gamma+1)}$ is a non-zero solution of the face equations of $f^{(\gamma+1)}$. We set

$$
\varphi_{\gamma+1, i}=\sum_{\gamma^{\prime} \leq \gamma+1} \psi_{\gamma^{\prime}}
$$

For a limit transfinite $\gamma$, we set

$$
\varphi_{\gamma, i}=\sum_{\gamma^{\prime}<\gamma} \psi_{\gamma^{\prime}} .
$$

This completes the description of the inductive process.

\subsection{Properties of the Series Expansions}

We assume, for a series we are attempting to build, that we were able to carry out the entire inductive process described above. So, at every step, we could find a decomposable face of the next Minkowski sum which satisfied the three criteria in Section 3.2. In Section 4 we 
describe conditions under which we can assure that the above process can be continued. Note, however, that these proofs are independent. In other words, when we attach the conditions we do not rely on any of the following properties in the construction. This discussion is more or less given as a general theorem on such series expansions. The series we build in the next section will have much stronger properties.

Theorem 3.1. Assume that the series expansions $\varphi_{i}$ have been constructed by the transfinitely inductive process described above. Then:

(a) The $\left\{\varphi_{i}\right\}$ are formal roots of the original system equations $\left\{F_{i}=0\right\}$.

(b) The exponents of the $\varphi_{i}$ lie in some strongly convex rational polyhedral cone $C$ in $\mathbb{R}^{\ell}$ such that $w \in C^{*}$.

(c) The exponents of the $\varphi_{i}$ lie in some lattice $(1 / N) \mathbb{Z}^{\ell}$.

Notice that (b) and (c) imply that the $\varphi_{i}$ are elements of the ring $\mathbb{C}\left(\left(C_{\mathbb{Q}}\right)\right)$, the ring of power series with support in some translate of $C$. This gives us that the power series have convergence in some translate of the cone $C^{*}$, by Theorem 2.7. Therefore, they have a common domain of convergence. Note that (c) also implies that the induction will never actually reach a transfinite state in the construction of these series. Unfortunately, the proof of (c) depends heavily on part (a) which depends on the existence of the $\varphi_{i}$. Thus transfinite induction is needed as a formal part of the construction to derive the properties of the series expansions, but is never needed in practice.

We prove this theorem in Section 3.6, but first we need to establish some technical facts. To prove part (a) we need to show that the largest terms of

$$
F_{i}\left(x_{1}, \ldots, x_{\ell}, \varphi_{\gamma, 1}, \ldots, \varphi_{\gamma, k}\right)
$$

with respect to the linear functional $w$ decrease as $\gamma$ increases, and that they decrease without bound. Notice that

$$
\begin{aligned}
F_{i}^{(\gamma+1)}(x, y) & =F_{i}^{(\gamma)}\left(x, c^{(\gamma)} x^{-\delta^{(\gamma)}}+y\right) \\
& =F_{i}^{(\gamma-1)}\left(x, c^{(\gamma)} x^{-\delta^{(\gamma)}}+c^{(\gamma-1)} x^{-\delta^{(\gamma)}}+y\right) \\
& =\cdots=F_{i}\left(x, y+\sum_{\gamma^{\prime} \leq \gamma} c^{\left(\gamma^{\prime}\right)} x^{-\delta^{\left(\gamma^{\prime}\right)}}\right) \\
& =F_{i}\left(x, \varphi_{\gamma}+y\right)
\end{aligned}
$$

and so

$$
F_{i}\left(x_{1}, \ldots, x_{\ell}, \varphi_{\gamma, 1}, \ldots, \varphi_{\gamma, k}\right)=F_{i}^{(\gamma+1)}\left(x_{1}, \ldots, x_{\ell}, 0, \ldots, 0\right) .
$$

Therefore we can prove (a) by showing that the maximal terms of $P_{i}^{(\gamma)} \cap \mathbb{R}^{\ell}$, with respect to $w$, decrease without bound. This, we accomplish by finding a sequence of supporting $w$-constant hyperplanes for $P_{i}^{(\gamma+1)}$, whose interesections with $\mathbb{R}^{\ell}$ decrease with respect to $w$. 


\subsection{Finding a Supporting Hyperplane}

Assume that

$$
F_{i}^{(\gamma)}(x, y)=\sum_{\alpha, \beta \in S_{i}^{(\gamma)}} a_{i, \alpha, \beta}^{(\gamma)} x^{\alpha} y^{\beta} .
$$

Explicitly carrying out the above substitution gives us

$$
\begin{aligned}
F_{i}^{(\gamma+1)}(x, y) & =\sum_{(\alpha, \beta) \in S_{i}^{(\gamma)}} a_{i, \alpha, \beta}^{(\gamma)} x^{\alpha}\left(c_{1}^{(\gamma)} x^{-\delta_{1}^{(\gamma)}}+y_{1}\right)^{\beta_{1}} \cdots\left(c_{k}^{(\gamma)} x^{-\delta_{k}^{(\gamma)}}+y_{k}\right)^{\beta_{k}} \\
& =\sum_{(\alpha, \beta) \in S_{i}^{(\gamma)}} a_{i, \alpha, \beta}^{(\gamma)} x^{\alpha} \prod_{j=1}^{k}\left(\sum_{v_{j}=0}^{\beta_{j}}\left(\begin{array}{c}
\beta_{j} \\
v_{j}
\end{array}\right)\left(c_{j}^{(\gamma)}\right)^{v_{j}} x^{-v_{j} \delta_{j}^{(\gamma)}} y_{j}^{\beta_{j}-v_{j}}\right) \\
& =\sum_{(\alpha, \beta)} \sum_{v}\left(\begin{array}{l}
\beta \\
v
\end{array}\right) a_{i, \alpha, \beta}^{(\gamma)}\left(c^{(\gamma)}\right)^{v} x^{\alpha-v_{1} \delta_{1}^{(\gamma)}-\cdots-v_{k} \delta_{k}^{(\gamma)}} y^{\beta-v} .
\end{aligned}
$$

Where $\left(\begin{array}{l}\beta \\ v\end{array}\right)=\Pi\left(\begin{array}{l}\beta_{i} \\ \nu_{i}\end{array}\right)$ is the product of the binomial coefficients.

We set $\left\langle\nu, \delta^{(\gamma)}\right\rangle=v_{1} \delta_{1}^{(\gamma)}+\cdots+v_{k} \delta_{k}^{(\gamma)}$ to simplify this expression, and we consolidate the coefficient, obtaining

$$
F_{i}^{(\gamma)}(x, y)=\sum_{(\alpha, \beta), \nu} A_{i, \alpha, \beta}^{\gamma, v} \cdot x^{\alpha-\left\langle v, \delta^{(\gamma)}\right\rangle} \cdot y^{\beta-\nu} .
$$

Analyzing the exponents appearing in this equation yields that the points in the polytope, $P_{i}^{(\gamma+1)}$, arise from points on $P_{i}^{(\gamma)}$ and lie on $k$-planes through these points with slope $\delta^{(\gamma)}$ with respect to $\mathbb{R}^{k}$, i.e. $k$-planes parallel to the face $f^{(\gamma)}$.

Consider the points in $P_{i}^{(\gamma+1)}$ that arise from $(\alpha, \beta) \in S_{i}^{(\gamma)}$. Since $v$ in the above expression ranges over the set

$$
\mathcal{Q}_{\alpha, \beta}=\left\{\left(v_{1}, \ldots, v_{k}\right): v_{i} \in \mathbb{Z}, 0 \leq v_{i} \leq \beta_{i} \text { for all } i\right\}
$$

and the exponent of $y$ is $\beta-v$, we see that points in $P_{i}^{(\gamma+1)}$ that arise from $(\alpha, \beta)$ project to points in the set $\mathcal{Q}_{\alpha, \beta}$ under the projection to the last $k$ coordinates. See Fig. 2.

Recall that in the inductive hypothesis we assumed that we had obtained faces $f_{i}^{(\gamma)}$ of $P_{i}^{(\gamma)}$ of dimension $>0$ and a $k$-face $f^{(\gamma)}$ of $P^{(\gamma)}$ such that

$$
f_{1}^{(\gamma)}+\cdots+f_{k}^{(\gamma)}=f^{(\gamma)} .
$$

As before, let $Q^{(\gamma)}$ be the $k$-plane containing $f^{(\gamma)}$ and let $Q_{i}^{(\gamma)}$ be the translate of $Q^{(\gamma)}$ which contains $f_{i}^{(\gamma)}$.

Lemma 3.2. Let $H_{i}^{(\gamma)}$ and $H^{(\gamma)}$ be the unique w-constant hyperplanes through the $k$ planes $Q_{i}^{(\gamma)}$ and $Q^{(\gamma)}$, respectively. Then $H_{i}^{(\gamma)}$ supports $P_{i}^{(\gamma+1)}$ and $H^{(\gamma)}$ supports $P^{(\gamma+1)}$. 


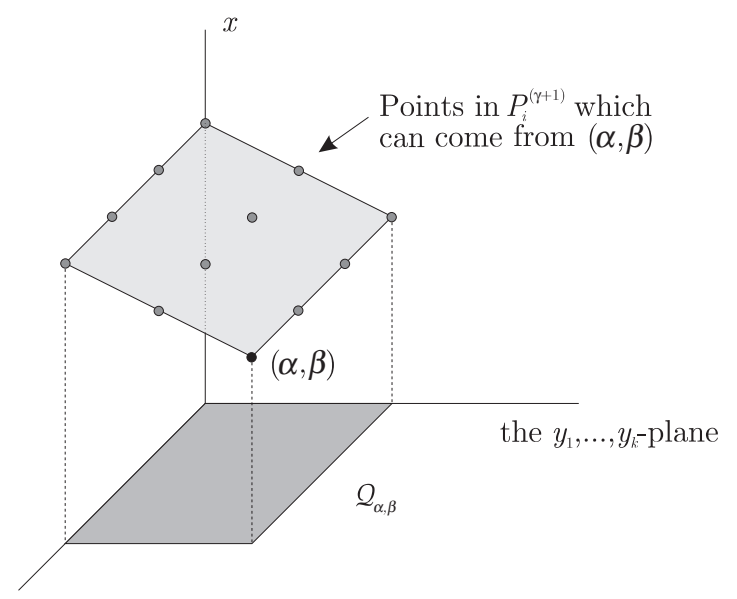

Fig. 2. Points on $P_{i}^{(\gamma+1)}$.

Proof. Note that these hyperplanes support their corresponding polytopes $P_{i}^{(\gamma)}$ and $P^{(\gamma)}$. We need to prove something slightly stronger, namely that they also support the next polytope in the sequence.

By the above discussion, we see that on the plane $Q_{i}^{(\gamma)}$ we get points in $P_{i}^{(\gamma+1)}$ whose coefficients are only affected by the terms of $f_{i}^{(\gamma)}=Q_{i}^{(\gamma)} \cap P_{i}^{(\gamma)}$. Moreover, we get that these points must lie in the region of $Q_{i}^{(\gamma)}$ lying above the sets $\mathcal{Q}_{\alpha, \beta}$ for the various $(\alpha, \beta) \in f_{i}^{(\gamma)}$. Note also that, since every point of $P_{i}^{(\gamma+1)}$ lies on a plane through some point of $P_{i}^{(\gamma)}$ parallel to $Q_{i}^{(\gamma)}$ and since $H_{i}^{(\gamma)}$ was a supporting hyperplane for $P_{i}^{(\gamma)}$, we see that $H_{i}^{(\gamma)}$ is a supporting hyperplane of $P_{i}^{(\gamma+1)}$. (Actually, to conclude this we also need that $P_{i}^{(\gamma+1)} \cap Q_{i}^{(\gamma)} \neq \emptyset$ so that the polytope in question actually touches this hyperplane. This is demonstrated in Remark 3.1.)

\subsection{Points in $\mathbb{R}^{\ell}$ Lie Strictly Below the Plane}

In order to show that the upper bound, with respect to $w$, on the terms in the series decreases at every step, we must prove the following lemma.

Lemma 3.3. The points of $P_{i}^{(\gamma+1)}$ which lie in the null-hyperplane lie strictly below the supporting hyperplane we constructed in Lemma 3.2. (Note that, in our view, the hyperplane supports from above, with respect to $w$.)

Proof. Let $q_{i}=Q_{i}^{(\gamma)} \cap \mathbb{R}^{\ell}$. We need to determine the coefficient of the monomial corresponding to $q_{i}$ in (3.21). Considering all the terms in (3.21) that contribute to the term corresponding to $q_{i}$, we get that this coefficient is equal to

$$
\sum_{(\alpha, \beta) \in S_{i}^{(\gamma)} \cap Q_{i}^{(\gamma)}} a_{i, \alpha, \beta}^{(\gamma)}\left(c^{(\gamma)}\right)^{\beta}
$$


Since this is precisely the $i$ th face equation of $f^{(\gamma)}$, and $c^{(\gamma)}$ was assumed to be a root of the face equations, we see that this coefficient is zero for all $i$.

Hence, if there are points of $P_{i}^{(\gamma)}$ on $\mathbb{R}^{\ell}$ they must lie "below" the $w$-constant hyperplane $H_{i}^{(\gamma)}$ in the sense that they have smaller weight than the points on the intersection of the hyperplane with $\mathbb{R}^{\ell}$. (All these points have the same weight by the definition of $w$-constant.)

Remark 3.1. There are several other points that we can explicitly determine on $P_{i}^{(\gamma+1)}$. Suppose $q$ is a point on $f_{i}^{(\gamma)}$ such that for every other point $q^{\prime}$ on $f_{i}^{(\gamma)}$ there is some $j$ such that $q_{j}>q_{j}^{\prime}$. Then the only term in $F_{i}^{(\gamma)}$ that can contribute to the term corresponding to $q$ in $F_{i}^{(\gamma+1)}$ is the term corresponding to $q$ itself. Therefore the coefficient of $q$ in $P_{i}^{(\gamma+1)}$ is unchanged from the previous step. Such vertices are called extreme on the face $f$. Note that this proves that there are points of $P_{i}^{(\gamma+1)}$ on $Q_{i}^{(\gamma)}$, and so $H_{i}^{(\gamma)}$ is a supporting hyperplane for $P_{i}^{(\gamma+1)}$.

\subsection{Proof of Theorem 3.1}

We begin with part (a). By Lemma 3.2, the polytope $P_{i}^{(\gamma+1)}$ is supported by $H_{i}^{(\gamma)}$ and by $H_{i}^{(\gamma+1)}$. Therefore, the following are upper bounds on the points of $P_{i}^{(\gamma+1)} \cap \mathbb{R}^{\ell}$ :

$$
\left\langle w, H_{i}^{(\gamma)} \cap \mathbb{R}^{\ell}\right\rangle \quad \text { and } \quad\left\langle w, H_{i}^{(\gamma+1)} \cap \mathbb{R}^{\ell}\right\rangle .
$$

Recall that $w$ is constant on $H_{i}^{(\gamma)}$ over every point of $\mathbb{R}^{k}$. We will have a decreasing upper bound on the points of $P_{i}^{(\gamma+1)}$ in $\mathbb{R}^{\ell}$ if we can show that

$$
\left\langle w, H_{i}^{(\gamma)} \cap \mathbb{R}^{\ell}\right\rangle>\left\langle w, H_{i}^{(\gamma+1)} \cap \mathbb{R}^{\ell}\right\rangle .
$$

Suppose that $\left\langle w, H_{i}^{(\gamma)} \cap \mathbb{R}^{\ell}\right\rangle \leq\left\langle w, H_{i}^{(\gamma+1)} \cap \mathbb{R}^{\ell}\right\rangle$. Since the slopes of $f^{(\gamma+1)}$ are all strictly greater than the corresponding slopes of $f^{(\gamma)}$, and $H_{i}^{(\gamma+1)}$ contains points of $P_{i}^{(\gamma+1)}$, we get that $H_{i}^{(\gamma+1)}$ lies strictly below $H_{i}^{(\gamma)}$ for all $i$. However, this contradicts the known fact that each of these hyperplanes support $P_{i}^{(\gamma+1)}$, demonstrated in Section 3.2.

The fact that this upper bound decreases without bound as $\gamma$ increases, follows from the fact that we are using a transfinitely inductive process. This process does not stop until the system $\varphi_{\gamma, i}$ satisfies the system of equations, i.e. until all the $P_{i}^{(\gamma)}$ have no terms left in $\mathbb{R}^{\ell}$. This proves that the series expansions obtained are formal roots of the initial system of equations.

Both (b) and (c) can be proved using an application of the single equation construction detailed in [8]. We accomplish this by considering resultants. Namely, consider that the $\varphi_{i}$ all have terms that are well ordered by the linear functional $w$. Therefore $\varphi_{i}$ are elements of the ring $\mathbb{C}((w))$ for every $i$, where $\mathbb{C}((w))$ is the ring of transfinite power series whose terms are well ordered with respect to $w$.

Let $F(x, y)$ and $G(x, y)$ be any polynomials, we denote by

$$
\mathcal{R}_{x_{j}}(F, G)
$$


the classical resultant of $F$ and $G$ with respect to $x_{j}$. This is a polynomial on the coefficients of $F$ and $G$ as polynomials in $x_{j}$, which is zero if and only if $F$ and $G$ have a common root. Note that in this case, the coefficients of $F$ and $G$ are themselves polynomials in several variables.

Since $\varphi_{1}, \ldots, \varphi_{k}$ simultaneously satisfy the system

$$
F_{i}(x, y)=F_{i}\left(y_{1}, \ldots, y_{k}\right)=0,
$$

we see that $\varphi_{1}, \ldots, \varphi_{k-1}$ satisfies the system of $k-1$ equations

$$
\mathcal{R}_{x_{k}}\left(F_{i}, F_{k}\right) \text {. }
$$

By repeated applications of this argument we see that $y_{1}=\varphi_{1}$ satisfies a polynomial $F$ in the variables $x_{1}, \ldots, x_{\ell}, y_{1}$. Therefore, since the terms of $\varphi_{1}$ are well ordered with respect to $w$ we see that $\varphi_{1}$ must be one of the series solutions to $F$ obtained by our main theorem in [8]. Therefore $\varphi_{1}$ has exponents lying in some lattice and contained in some strongly convex rational polyhedral cone $C$ such that $w \in C^{*}$. The proofs for the other $\varphi_{i}$ follow similarly.

\section{Conditions on the Roots of the Face Equations}

In this section we explore one situation in which we can continue the above process. In this case we can determine a great deal about the structure of the series solutions generated by the procedure. We require that the initial choice of roots of the face equations be a simple solution of the system, i.e. the Jacobian of the face equations is non-zero at $c$, and that all partial derivatives of the face equations of the initial face do not vanish at $c$.

Theorem 4.1. Let $P$ be the Minkowski sum of the polytopes $P_{i}$ of the polynomials $F_{i}$, and let $\delta$ be the matrix of slopes of $f$. Assume that the root $c=\left(c_{1}, \ldots, c_{k}\right)$ of the face equations $f$ is a simple root, and assume that the first partial derivatives of these face equations are all non-zero at $c$. Then:

(a) The induction of Section 3.2 can always be continued.

(b) The support of $\varphi_{i}$ lies in the translate of the barrier cone of $f$ with vertex at $-\delta_{i}$ for all $i$ and (Theorem 2.7) the $\varphi_{i}$ converge in $\log ^{-1}\left(C^{*}+v\right)$ for some $v$ in $\left(\mathbb{R}^{\ell}\right)^{*}$.

The proof of this theorem is in Section 4.2, but first we need to analyze the second step of the induction for such systems.

Recall that $f^{(0)}$ was the chosen face of the Minkowski sum of the initial polytopes, and $f_{1}^{(0)}, \ldots, f_{k}^{(0)}$ were the faces that sum to $f^{(0)}$. We had

$$
\sum_{(\alpha, \beta) \in S_{i}^{(0)} \cap Q_{i}^{(0)}} a_{i, \alpha, \beta}^{(0)} c^{\beta}=0
$$

as the face equations of $f$, where $Q^{(0)}$ was the $k$-plane determined by $f^{(0)}$. Further, $Q_{i}^{(0)}$ was the translate of $Q^{(0)}$ containing $f_{i}^{(0)}$. 
We now replace $c$ by $t=\left(t_{1}, \ldots, t_{k}\right)$ in (4.1), i.e. we consider the expressions on the left-hand side of (4.1) as polynomials $E_{i} \in \mathbb{C}\left[t_{1}, \ldots, t_{n}\right]$ :

$$
E_{i}\left(t_{1}, \ldots, t_{k}\right)=\sum_{(\alpha, \beta) \in S_{i} \cap Q_{i}^{(0)}} a_{i, \alpha, \beta} t^{\beta} .
$$

The condition that $c$ is a simple root of (4.1) means that the matrix

$$
\mathcal{M}=\left[\begin{array}{ccc}
\frac{\partial E_{1}}{\partial t_{1}}(c) & \ldots & \frac{\partial E_{1}}{\partial t_{k}}(c) \\
\vdots & \ddots & \vdots \\
\frac{\partial E_{k}}{\partial t_{1}}(c) & \ldots & \frac{\partial E_{k}}{\partial t_{k}}(c)
\end{array}\right]
$$

is non-singular, i.e. $\operatorname{det}(\mathcal{M})$ is non-zero.

Our second assumption gives that for all $i$ and $j$,

$$
\frac{\partial E_{i}}{\partial t_{j}}(c) \neq 0 .
$$

To draw conclusions from this second assumption we need to introduce some notation.

Definition 4.1. Let $P(f)=\operatorname{conv}\left\{p_{1}, \ldots, p_{n}\right\}$ be any $m$-dimensional Newton polytope. For each of these points, set $p_{i}=\left(p_{i, 1}, \ldots, p_{i, m}\right)$. We define the partial derivative of the $P$ with respect to $t_{i}$ to be

$$
P\left(\frac{\partial F}{\partial t_{j}}\right)=\frac{\partial P(F)}{\partial t_{j}} .
$$

Thus, taking the derivative of a polytope with respect to $t_{j}$ has the effect of shifting it by -1 in the $t_{j}$ direction, and removing the parts that have negative $t_{j}$ coordinate.

\subsection{Finding an Appropriate Decomposable Face on $P^{(1)}$}

We need to find a decomposable admissible face of the polytope $P^{(1)}$ explicitly. In fact, each polytope $P_{i}^{(1)}$ contains an extremely simple admissible face which sums to the desired face of $P^{(1)}$.

Lemma 4.2. Under the assumptions of Theorem 4.1, one of the $P_{i}^{(1)}$ has a $k$-face whose vertices have the form

$$
\begin{aligned}
r_{i} & =\left(r_{i 1}, \ldots, r_{i \ell}, 0, \ldots, 0\right), \\
s_{i}^{(1)} & =\left(s_{i 1}^{(1)}, \ldots, s_{i \ell}^{(1)}, 1,0, \ldots, 0\right), \\
& \vdots \\
s_{i}^{(k)} & =\left(s_{i 1}^{(k)}, \ldots, s_{i 1}^{(k)}, 0, \ldots, 0,1\right) .
\end{aligned}
$$


Furthermore, each of the other $P_{i}^{(1)}$ has a $(k-1)$-face spanned by vertices of the form $s_{i}^{(1)}, \ldots, s_{i}^{(k)}$.

Proof. Let

$$
q_{i}=Q_{i}^{(0)} \cap \mathbb{R}^{\ell}
$$

be the intersection points of these $k$-planes with $\mathbb{R}^{\ell}$. We noticed, in the second step of the construction, that since this coefficient is the same as the $i$ th-face equation, the coefficient on the term corresponding to $q_{i}$ in $F_{i}^{(1)}$ is zero for all $i$. Let

$$
q_{i}^{(j)}=\frac{\partial Q_{i}^{(0)}}{\partial t_{j}} \cap \mathbb{R}^{\ell}
$$

The Newton polytope of the partial derivative is just a translation of the original Newton polytope (with part cut off at the null-hyperplane). So, showing that a point lying over $e_{i}$ is actually a vertex on the bottom of the polytope is equivalent to showing that the coefficient on the term corresponding to $q_{i}^{(j)}$ in $\partial F_{i}^{(1)} / \partial y_{j}$ is non-zero. (Such a point would necessarily lie on the previous hyperplane, but points in the null-hyperplane lie below it with respect to $w$.)

By the chain rule, we get that

$$
\frac{\partial}{\partial y_{j}}\left(F_{i}^{(1)}\right)=\left(\frac{\partial}{\partial y_{j}}\left(F_{i}^{(0)}\right)\right)\left(x, y+c^{(\gamma)} x^{-\delta^{(\gamma)}}+y_{k}\right) .
$$

Therefore, the partial derivatives of face equations $E_{i}$ of $f^{(0)}$ are the face equations of the partial derivatives of $f^{(0)}$. So, in the same way that we showed that the coefficient on $x^{q_{i}}$ in $F_{i}^{(1)}$ was zero, we can show that the coefficient on

$$
(x, y)^{q_{i}^{(j)}} \text { in } \frac{\partial F_{i}^{(1)}}{\partial y_{j}} \text { is } \frac{\partial E_{i}}{\partial t_{j}}(c) .
$$

Since

$$
\frac{\partial E_{i}}{\partial t_{j}}(c) \neq 0
$$

for all $i$ and $j$, we see that the term in $\partial F_{i}^{(1)} / \partial y_{j}$ corresponding to $q_{i}^{(j)}$ has a nonzero coefficient. Let $e_{j}$ be the $j$ th coordinate vector in $\mathbb{R}^{k}$, and for all $i$ let $s_{i}^{(j)}=$ $\left(s_{i 1}^{(j)}, \ldots, s_{i k}^{(j)}, e_{j}\right)$ be the point on $Q_{i}^{(0)}$ that projects to the point $\left(0, e_{j}\right)$ in $\mathbb{R}^{k}$. Taking the partial derivative of $P_{i}^{(1)}$ with respect to $y_{i}$ takes $s_{i}^{(j)}$ to $q_{i}^{(j)}$. By (4.9), and the fact that we are taking the derivative of a monomial containing $t_{i}^{1}$, we see that the coefficient on

$$
(x, y)^{s_{i}^{(j)}} \text { in } F_{i}^{(1)} \text { is } \frac{\partial E_{i}}{\partial t_{j}}(c) .
$$

Hence the term in $F_{i}^{(1)}$ corresponding to $s_{i}^{(j)}$ has a non-zero coefficient for all $j$. So $s_{i}^{(j)}$ is contained in, and is in fact a vertex in, $P_{i}^{(1)}$. 

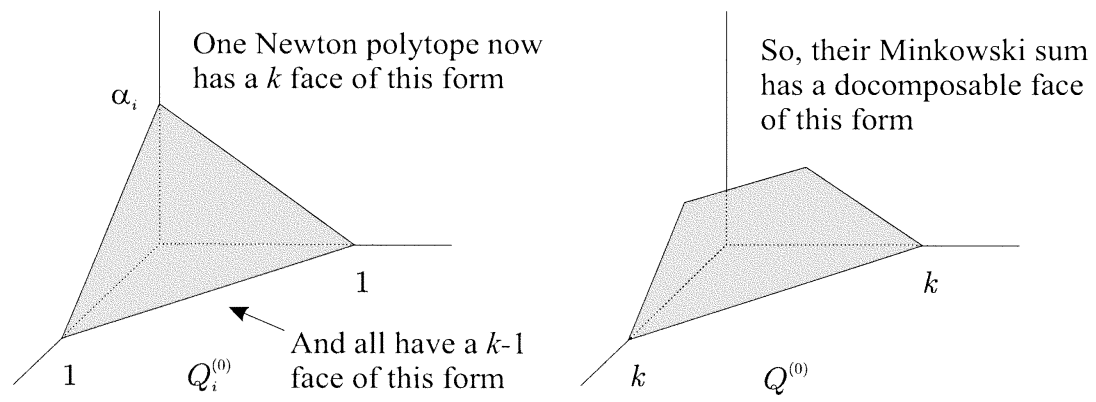

Fig. 3. The face on the Minkowski sum of the new polytopes.

Let $r_{i}=\left(r_{i 1}, \ldots, r_{i \ell}, 0, \ldots, 0\right)$ be the maximal point of $P_{i}^{(1)}$ in $\mathbb{R}^{\ell}$, with respect to $w$. If we assume that $\left\{y_{i}=c_{i}^{(0)} x^{\delta_{i}^{(0)}}\right\}$ is not a solution to all of the equations, then there must be points of $P_{i}^{(1)}$ on $\mathbb{R}^{\ell}$ for some $i$. If $P_{i}^{(1)}$ has no points in $\mathbb{R}^{\ell}$, then we formally set $r_{i}=\infty$. We add the finite $r_{i}$ 's to the $s_{i}^{(j)}$ 's, and so get a set of points on $P^{(1)}$. To complete this construction we must use these points to find an explicit decomposable $k$-face on $P^{(1)}$. The structure of the slopes of this face give us some interesting results on the structure of the fractional power series that we generate from this process.

First, we notice that, on each of the $P_{i}^{(1)}$, the $(k-1)$-simplex formed by the points $\left\{s_{i}^{(j)}\right\}$ is a $(k-1)$-face. (Call this face $\tilde{f}_{i}$.) This is because this $(k-1)$-simplex is contained in $Q_{i}^{(0)}$, a supporting $k$-plane for $P_{i}^{(1)}$, and because each of these points lies over one of the coordinate axes. (There are no points of $P_{i}^{(1)}$ with negative $y_{j}$ for any $j$.) Therefore all of the $P_{i}^{(1)}$ have at least one parallel $(k-1)$-face. Moreover, this face is maximized by $w$ since $Q_{i}^{(0)}$ is a supporting hyperplane in the direction of $w$. Therefore, $\tilde{f}_{1}+\cdots+\tilde{f}_{k}$ is a $(k-1)$-face of $P^{(1)}$. This face has vertices with the $\alpha_{i}$ coordinate equal to $k e_{i}$ for all $i$, as shown in Fig. 3.

Consider, next, the points $r_{i}$. In the discussion above, we showed that $\left\langle w, r_{i}\right\rangle<$ $\left\langle w, q_{i}\right\rangle$. (Recall that $q_{i}=Q_{i}^{(0)} \cap \mathbb{R}^{\ell}$.) Choose $i_{0}$ such that the expression

$$
\left\langle w, q_{i_{0}}\right\rangle-\left\langle w, r_{i_{0}}\right\rangle
$$

is minimal. The $k$-face formed by

$$
\left\{s_{i_{0}}^{(1)}, \ldots, s_{i_{0}}^{(k)}, r_{i_{0}}\right\}
$$

is a $w$-maximal face of $P_{i_{0}}^{(0)}$. Call this face $f_{i}$. Reorder the polynomials (and hence the polytopes) so that the first $i^{\prime}$ polytopes satisfy the above minimality condition, i.e. for all $i>i^{\prime}$ we have that

$$
\left\langle w, q_{i}\right\rangle-\left\langle w, r_{i}\right\rangle>\left\langle w, q_{i^{\prime}}\right\rangle-\left\langle w, r_{i^{\prime}}\right\rangle
$$

Then $f^{(1)}:=f_{1}+\cdots+f_{i^{\prime}}+\tilde{f}_{i^{\prime}+1}+\cdots+\tilde{f}_{k}$ is a maximal admissible decomposable face of $P^{(1)}$. For the sake of notation rename its summands as $f_{i}^{(1)}$. 
Consider the parallel faces $f_{1}^{(1)}, \ldots, f_{i^{\prime}}^{(1)}$. We see that $f_{i}^{(1)}$ is spanned by $k+1$ affinely independent points

$$
\begin{aligned}
r_{i} & =\left(r_{i 1}, \ldots, r_{i \ell}, 0, \ldots, 0\right), \\
s_{i}^{(1)} & =\left(s_{i 1}^{(1)}, \ldots, s_{i \ell}^{(1)}, 1,0, \ldots, 0\right), \\
& \vdots \\
s_{i}^{(k)} & =\left(s_{i 1}^{(k)}, \ldots, s_{i 1}^{(k)}, 0, \ldots, 0,1\right)
\end{aligned}
$$

as required. Moreover, these are the only points of $\operatorname{Supp}\left(F_{i}^{(1)}\right)$ that $f_{i}^{(1)}$ contains. The same analysis holds for $i$ 's greater than $i^{\prime}$, but without the first point in the list. They are $(k-1)$-faces, and each of the previous $k$-faces contains such a $(k-1)$-face.

The $k$-faces summing to this face are all parallel, and the $(k-1)$-faces all lie on translates of $Q^{(0)}$. Therefore, the slopes of $f^{(1)}$ with respect to $x$ are the same as the slopes of $f_{1}^{(1)}$. So, consider the slope of $f_{1}^{(1)}$ in the direction of $y_{i} . f_{1}^{(1)}$ contains the point $s_{1}^{(i)}$, which lies over $e_{i}$. It also contains the point $r_{1}$ which lies over zero. These two points differ, over $\mathbb{R}^{k}$, by one in the $y_{i}$ direction. Therefore the slope of $f_{1}^{(1)}$ in the $y_{i}$ direction is

$$
\delta_{i}^{(1)}:=\left(s_{11}^{(i)}-r_{11}, \ldots, s_{1 k}^{(i)}-r_{1 k}\right)=s_{1}^{(i)}-r_{1} .
$$

We set the exponents in the second terms of our series expansions to be $-\delta^{(1)}$.

The next two lemmas prove Theorem 4.1 specifically for the second step in the construction. From there, we can complete the proof of the theorem by induction.

Lemma 4.3. Part (a) of Theorem 4.1 holds for the second step in construction as specified above.

Proof. By Lemma 4.2, the sum polytope $P^{(1)}$ has a decomposible face of the proper form. To finish the proof, we need to show that the face equations of $f^{(1)}$ have a solution. Suppose that the coefficient on $r_{i}$ is $\rho_{i}$. By (4.10) we get that the face equations of $f^{(1)}$ are

$$
\begin{aligned}
\frac{\partial E_{1}}{\partial t_{1}}(c) t_{1}+\cdots+\frac{\partial E_{1}}{\partial t_{k}}(c) t_{k} & =\rho_{1}, \\
& \vdots \\
\frac{\partial E_{k}}{\partial t_{1}}(c) t_{1}+\cdots+\frac{\partial E_{k}}{\partial t_{k}}(c) t_{k} & =\rho_{k} .
\end{aligned}
$$

Since the matrix $\mathcal{M}$ in (4.3) is non-singular and not all of the $\rho_{i}$ 's are zero, this system of equations has a unique solution.

Lemma 4.4. Part (b) of Theorem 4.1 holds for the second step in the construction.

Proof. To prove part (b) of this theorem, at least for this step, we need to show that for every $w^{\prime} \in B\left(f^{(0)}\right)$ and every $i$, we get that $-\delta_{i}^{(1)}$ is in the translate of the barrier cone 
of $f=f^{(0)}$ with vertex at $-\delta_{i}^{(0)}$. For this we follow an argument similar to the one in the corresponding lemma in [8].

Recall that $Q_{i}^{(0)}$ has the same slopes as $f^{(0)}$. Since $s_{1}^{(i)}$ is on $Q_{i}^{(0)}$, these slopes are given by

$$
\delta_{i}^{(0)}=\left(s_{11}^{(i)}-q_{11}, \ldots, s_{1 k}^{(i)}-q_{1 k}\right)=s_{1}^{(i)}-q_{1} .
$$

By (4.11) we see that

$$
\left\langle w, \delta_{i}^{(1)}\right\rangle=\left\langle w, s_{1}^{(i)}-r_{1}\right\rangle>\left\langle w, s_{1}^{(i)}-q_{1}\right\rangle=\left\langle w, \delta_{i}^{(0)}\right\rangle
$$

Therefore we have that

$$
\left\langle w,-\delta_{i}^{(1)}\right\rangle<\left\langle w,-\delta_{i}^{(0)}\right\rangle
$$

implying that, at least at this term, the series is decreasing with respect to $w$.

To show that the series is actually in the normal cone, we need to show that the inequality in (4.17) holds for all $w^{\prime} \in N\left(f^{(0)}\right)$. For all $w^{\prime} \in B\left(f^{(0)}\right)$, let $H_{w^{\prime}}$ be the unique $w^{\prime}$-constant hyperplane through $f_{i}$. Let $H_{i, w^{\prime}}$ be the translate of $H_{w^{\prime}}$ which supports $P_{i}^{(0)}$ at $f_{i}^{(0)}$. Then $P_{1}^{(0)}$ also supports $P_{i}^{(1)}$ since all points of $P_{i}^{(1)}$ are translates of points of $P_{i}^{(0)}$ in the direction of $f^{(0)}$. Therefore the argument used above for $w$ will also work for all $w^{\prime}$.

\subsection{Proof of Theorem 4.1}

We prove this theorem by induction. Lemmas 4.3 and 4.4 form the base case of the induction. So, suppose in previous steps we built the exponent $\delta^{(n-1)}$ and coefficient $c^{(n-1)}$. Also suppose that, in doing so, we have built $F_{i}^{(n-1)}$ and its polytope $P_{i}^{(n-1)}$ for all $i$. Suppose, further, that the $P_{i}^{(n-1)}$ satisfy the following condition:

- The points $s_{j}^{(i)}$, defined above for the second step of the construction, are vertices of $P_{i}^{(n-1)}$, and they are extreme vertices of $f_{i}^{(n-1)}$. (Extreme in the sense of Remark 3.1.) Note that this condition holds for the case $n=3$ by Lemma 4.2.

Since the $s_{j}^{(i)}$ are on a supporting hyperplane for $P_{i}^{(n-1)}$, and are extreme on $f_{i}^{(n-1)}$, by Remark 3.1 their coefficients are preserved in the transition from $P_{i}^{(n-1)}$ to $P_{i}^{(n)}$. Therefore we have, on the $P_{i}^{(n)}$, faces of the same form as above. Moreover, the face equations of these faces have the same coefficients as the system in (4.15). Since, by assumption, the approximation from the $(n-1)$ st step of the induction did not satisfy all the equations, some of the $\rho_{i}$ are again non-zero. So we can continue the induction.

Since the faces we obtained for $P_{i}^{(n)}$ have the same form as those in the second step, the same arguments apply to show that the support of the series lies in a translate of the barrier cone of $f^{(0)}$ with vertex at $-\delta_{i}^{(0)}$. (Since $P_{i}^{(n)}$ lies in the barrier wedge of $f^{(0)}$, and the points of $P_{i}^{(n+1)}$ are obtained by translating points in $P_{i}^{(n)}$ in the direction of $f^{(n)}$, we see that $P_{i}^{(n+1)}$ is also in this barrier wedge.) 


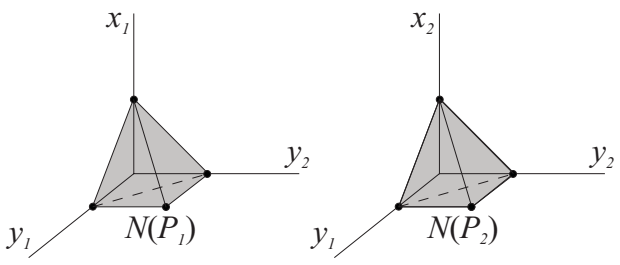

Fig. 4. The Newton polytopes of $P_{1}$ and $P_{2}$.

\subsection{An Example}

To get a feeling for the actual mechanics of this construction, we work out the first few steps in the following system:

$$
\begin{aligned}
& P_{1}=x_{1}+y_{1}-2 y_{2}+y_{1} y_{2}=0, \\
& P_{2}=x_{2}-y_{1}+y_{2}+2 y_{1} y_{2}=0 .
\end{aligned}
$$

The Newton polytopes $N\left(P_{1}\right)$ and $N\left(P_{2}\right)$ are show in Fig. 4. Notice that each is a threedimensional polytope since $P_{1}$ does not have any terms involving $x_{2}$, and likewise for $P_{2}$ and $x_{1}$.

To carry out the construction, we need to choose a linear functional to give relative weights to monomials in the $x_{i}$. So, let

$$
w=(1, \pi / 2) \approx(1,1.57)
$$

Recall that the coordinates of $w$ should be linearly independent over the rationals to give a well ordering on the set of monomials. For the first step in the construction, this functional $w$ chooses the lower triangular face $f_{1}=\{(1,0,0,0),(0,1,0,0),(0,0,1,0)\}$ from $N\left(P_{1}\right)$, and one-dimensional face $f_{2}=\{(1,0,0,0),(0,1,0,0)\}$ from $N\left(P_{2}\right)$. Taking the Minkowski sum, yields the face on the sum polytope whose vertices are $\{(2,0,0,0),(0,2,0,0),(1,0,1,0),(0,1,0,0)\}$, as shown in Fig. 5.

The slope matrix of $f_{1}+f_{2}$ is

$$
\delta=\left[\begin{array}{ll}
-1 & 0 \\
-1 & 0
\end{array}\right]
$$

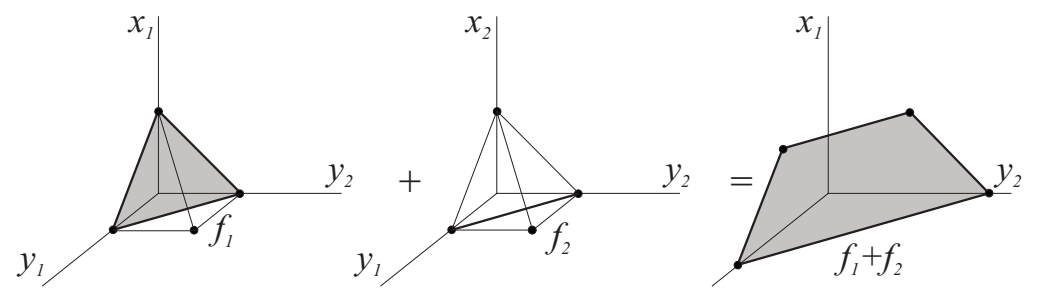

Fig. 5. The faces for the first step. 
since it lies entirely within the hyperplane $x_{2}=0$. Therefore, our first term will be of the form

$$
\begin{aligned}
& y_{1}=a x_{1}+\text { h.o.t. } \\
& y_{2}=b x_{1}+\text { h.o.t. }
\end{aligned}
$$

We find $a$ and $b$ by solving the face equations given by

$$
\begin{aligned}
1+a-2 b & =0, \\
-a+b & =0,
\end{aligned}
$$

which has a single solution, $a=b=1$. Therefore, the expansions are

$$
\begin{aligned}
& y_{1}=x_{1}+\text { h.o.t. } \\
& y_{2}=x_{1}+\text { h.o.t. }
\end{aligned}
$$

always remembering that h.o.t. means terms that are greater with respect to the chosen functional $w$.

The second term can be obtained similarly once we substitute the expressions in (4.23) into our original system in (4.18) to obtain the system

$$
\begin{aligned}
y_{1}-2 y_{2}+x_{1}^{2}+x_{1} y_{1}+x_{1} y_{2}+y_{1} y_{2} & =0, \\
x_{2}-y_{1}+y_{2}+2 x_{1}^{2}+2 x_{1} y_{1}+2 x_{1} y_{2}+2 y_{1} y_{2} & =0 .
\end{aligned}
$$

At this point, the second polytope becomes four-dimensional, and we use the functional $w$ to visualize it and all of the polytopes from here on out. Consider that $w$ yields a well ordering on the monomials in the $\left(x_{1}, x_{2}\right)$-plane, and therefore we can collapse the $\left(x_{1}, x_{2}\right)$-plane to a line in our diagram with the projection

$$
\pi(i, j)=w \cdot(i, j) .
$$

Since this preserves the ordering of the monomials, it preserves the lowest faces with regard to the projection to the $\left(y_{1}, y_{2}\right)$-plane. The resulting polytopes are shown in Fig. 6 from which we obtain the terms $a x_{2}$ and $b x_{2}$ and the face equations

$$
\begin{aligned}
a-2 b & =0, \\
1-a+b & =0 .
\end{aligned}
$$

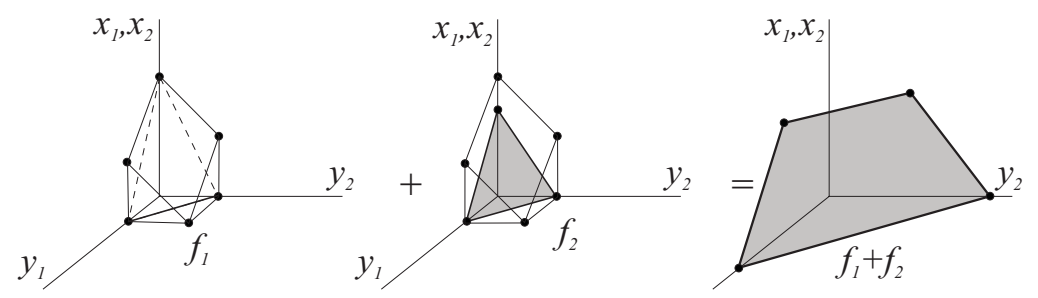

Fig. 6. The polytopes and faces for the second step. 
These equations have the single solution $a=\frac{2}{3}$ and $b=\frac{1}{3}$. So the second stage of the expansion is

$$
\begin{aligned}
& y_{1}=x_{1}+\frac{2}{3} x_{2}+\text { h.o.t. } \\
& y_{2}=x_{1}+\frac{1}{3} x_{2}+\text { h.o.t. }
\end{aligned}
$$

If we continue the construction for a few more terms, we obtain the series expansion

$$
\begin{aligned}
& y_{1}=x_{1}+\frac{2}{3} x_{2}+5 x_{1}^{2}+5 x_{1} x_{2}+\text { h.o.t. } \\
& y_{2}=x_{1}+\frac{1}{3} x_{2}+3 x_{1}^{2}+3 x_{1} x_{2}+\text { h.o.t. }
\end{aligned}
$$

\subsection{Complete Systems and Normal Cones}

The following corollary relates the maximal collections of series solutions to the normal cones of the faces involved in the constructions.

Corollary 4.5. Suppose that all of the decomposable faces in the w-maximal section of $P^{(0)}$ have face equations with maximal numbers of roots (i.e. the number of roots is equal to the mixed volume of the projection to $\mathbb{R}^{k}$ ). Also suppose that all the roots of these systems satisfy the conditions of Theorem 4.1. Then the number of solutions to the system $\left\{F_{i}\right\}$ in $\mathbb{C}\left(\left(C_{\mathbb{Q}}\right)\right)$, where

$$
C=\bigcup_{\substack{f \text { a face of } \mathcal{S}, f \text { decomposable }}} B(f)=\bigcup_{\substack{f \text { a face of } \mathcal{S}, f \text { decomposable }}} N^{*}(f)
$$

is equal to the mixed volume of the projection of the polytopes $P_{1}^{(0)}, \ldots, P_{k}^{(0)}$ :

$$
V=\operatorname{Vol}\left(\pi\left(P\left(F_{1}\right)\right), \ldots, \pi\left(P\left(F_{k}\right)\right)\right) .
$$

Therefore, the number of solutions converging in some translate of the intersection of the normal cones of these faces is equal to $V$.

Note that this intersection is non-empty since it contains $w$.

Proof. We have, by Theorem 4.1, at least one distinct series in this ring for each root. Therefore, this ring contains at least $V$ solutions. By Theorem 2.1 the number of series in this ring that satisfy the system can be no more than this mixed volume.

\section{Fiber Polytopes and the Mixed Fiber Polytope}

Suppose that all of the faces of $P$ satisfy the conditions of Corollary 4.5. Then this corollary tells us that complete systems of solutions correspond to maximal collections of decomposable, admissible faces whose normal cones have a non-trivial intersection. Consider that, by choosing a linear functional $w$, we get a coherent section of the projection

$$
\pi: P=P_{1}+\cdots+P_{k} \longrightarrow \mathbb{R}^{k}
$$


of the Minkowski sum of the Newton polytopes of the original equations. By changing $w$, we change the section. Note, however, that if two sections differ by only indecomposable faces, then they yield the same set of series solutions in the above construction. If they differ by any decomposable faces, then they give (at least some) distinct series solutions. Therefore, the maximal sets of series solutions of $F_{1}, \ldots, F_{k}$ correspond to equivalence classes of coherent sections of $\pi$, where two sections are equivalent if they contain the same sets of decomposable faces.

If $P_{1}=\cdots=P_{k}$, then $P$ is a dilation of $P_{i}$ for all $i$. Therefore, every face on $P$ is decomposable, and hence the maximal sets of series solutions correspond precisely to the coherent sections of $\pi$.

Let $\Delta$ be the fan in $\mathbb{R}^{\ell}$ whose cones are the domains of convergence of the maximal collections of series constructed above. Consider the fiber polytope [3] associated to the projection $\pi$. Let $Q$ be the image of $\pi$, then this fiber polytope is denoted by

$$
\Sigma_{\pi}(P)=\Sigma_{\pi}(P, Q)=\left\{\int_{Q} \gamma(x) d x: \gamma \in \Gamma(P)\right\} .
$$

Recall that the vertices of $\Sigma_{\pi}(P)$ are in one-to-one correspondence with the coherent sections of $\pi$, and that the barrier cone of the vertex $v_{w}$ is equal to the union of the barrier cones of the faces that comprise the section. Therefore we get the following corollary to Theorem 4.1.

Corollary 5.1. The normal fan $\Delta_{\Sigma}$ of $\Sigma_{\pi}(P)$ is a refinement of $\Delta$. If $P_{1}=\cdots=P_{k}$, then $\Delta_{\Sigma}=\Delta$.

The properties of the fan $\Delta$ suggests the existence of a polytope $\Sigma_{\pi}\left(P_{1}, \ldots, P_{k}\right)$ called the mixed fiber polytope of the polytopes $P_{i}$ associated to the projection $\pi$. This polytope should satisfy the following properties:

1. $\Sigma_{\pi}\left(P_{1}, \ldots, P_{k}\right)$ is a Minkowski summand of $\Sigma_{\pi}(P)$, and hence the normal fan of $\Sigma_{\pi}(P)$ is a refinement of the normal fan of $\Sigma_{\pi}\left(P_{1}, \ldots, P_{k}\right)$.

2. If $P_{1}=P_{2}=\cdots=P_{k}$, then $\Sigma_{\pi}\left(P_{1}, \ldots, P_{k}\right)=\Sigma_{\pi}(P)$.

It is hoped that a generalization of the mixed secondary polytopes introduced in [9] will yield the desired polytope. The relationship between the mixed fiber polytopes and mixed secondary polytopes should be analogous to the unmixed case.

\section{The Mixed Discriminant and Mixed Fiber Polytopes}

As in [8] these results indicate a relationship between the fiber polytope and a certain ramification locus. Namely, consider the ramification locus of the variety $X=\left\{F_{1}=\right.$ $\left.\cdots=F_{k}=0\right\}$. Assume that $X$ is smooth. Then this locus corresponds to all multiple points in the projection to the $\left(x_{1}, \ldots, x_{\ell}\right)$-hyperplane, and since $X$ cannot have singularities, must lie outside the domains where we have complete collections of series solutions.

Just as in [8], the Log of this ramification locus is bounded by the cones which contain maximal series solution sets. 
Theorem 6.1. If the Newton polytopes of the $F_{i}$ coincide, then the $\log$ of the ramification locus of the projection of $x$ to the $\left(x_{1}, \ldots, x_{\ell}\right)$-hyperplane is bounded by translates of the normal cones of the fiber polytope associated to the projection of the Minkowski sum of the $F_{i}$ to $\mathbb{R}^{k}$.

In general, it will be necessary to build the mixed fiber polytope to extend this relationship.

In [8] this ramification locus corresponded to the zero locus of the discriminant of $F$ with respect to $x_{N+1}$. Here, the locus is the zero locus of a generalization of this discriminant, called the mixed discriminant.

Intuitively, it is the locus where the equations $F_{i}=0$ have a common multiple root. Generically, $k$ equations in $k$ variables intersect in a finite number of roots determined by the mixed volume of their Newton polytopes. We wish to make explicit the conditions on the $F_{i}$ which determine when two of these roots merge into a common double root.

This happens when a translate of the $\left(y_{1}, \ldots, y_{k}\right)$-hyperplane is tangent to the variety $X$. However, this is equivalent to saying that the tangent planes to the varieties $\left\{F_{i}=0\right\}$ intersect in an $\ell$-plane which lies parallel to the $\left(y_{1}, \ldots, y_{k}\right)$-hyperplane. This in turn is equivalent to the condition that

$$
\operatorname{det}\left(\left[\begin{array}{ccc}
\frac{\partial F_{1}}{\partial y_{1}} & \cdots & \frac{\partial F_{1}}{\partial y_{k}} \\
\vdots & \ddots & \vdots \\
\frac{\partial F_{k}}{\partial y_{1}} & \cdots & \frac{\partial F_{k}}{\partial y_{k}}
\end{array}\right]\right)=0 .
$$

In other words, the $F_{i}$ vanish, and their Jacobian vanishes. This gives a codimension 1 condition on the space of all sections of $X$ over $x_{1}, \ldots, x_{\ell}$.

The above results give the following result on the zero locus of the mixed discriminant of $F_{1}, \ldots, F_{k}$.

Theorem 6.2. Suppose that the Newton polytopes of the $F_{i}$ coincide, and that their corresponding variety $X$ is smooth. Then the zero locus of the mixed discriminant of the $F_{i}$ with respect to the variables $y_{1}, \ldots, y_{k}$ is bounded by the normal cones of the fiber polytope associated to the projection of the Minkowski sum of the $F_{i}$ to $\mathbb{R}^{l}$.

\section{References}

1. M. Artin, On the solutions of analytic equations, Invent. Math., 5 (1968), 277-291.

2. D. N. Bernstein, The Number of roots of a system of equations, Functional Anal. Appl. 9 (1975), 183-185.

3. L. J. Billera, B. Sturmfels, Fiber polytopes, Ann. of Math., 135 (1992), 527-549.

4. A. Bruno, A. Soleev, The local uniformization of branches of an algebraic curve, Contemp. Math., 131(3) (1992), 361-378.

5. I. M. Gelfand, M. M. Kapranov, A. V. Zelevinsky, Discriminants, Resultants and Multidimensional Determinants, Birkhäuser, Boston, 1994.

6. F. Hausdorff, Set Theory, Chelsea, New York, 1957.

7. S. Kranz, Function Theory of Several Complex Variables, Wiley, New York, 1982.

8. J. McDonald, Fiber polytopes and fractional power series, J. Pure Appl. Algebra, 104 (1995), 213-233. 
9. T. Michiels, R. Cools, Decomposing the secondary Cayley polytope, Discrete Comput. Geom., 23 (2000), $367-380$.

10. B. Poonen, Maximally complete fields, Enseign. Math., 39 (1993), 87-106.

11. Z. Smilansky, Decomposability of polytopes and polyhedra, Geom. Dedicata, 24 (1987), 29-49.

12. D. Stefanescu, A method to obtain algebraic elements over $k((T))$ in positive characteristics, Bull. Math. Soc. Sci. R. S. Roumanie, 26(74) (1982), N1, 77-91.

13. D. Stefanescu, On meromorphic formal power series, Bull. Math. Soc. Sci.R. S. Roumanie, 27(75), (1983), N2, 1-10.

14. R. J. Walker, Algebraic Curves, Princeton University Press, Princeton, NJ, 1950.

15. G. M. Ziegler, Lectures on Polytopes, Springer-Verlag, New York, 1995.

Received January 31, 2000, and in revised form April 30, 2001. Online publication March 27, 2002. 